\title{
Infinitely many geometrically distinct solutions for periodic Schrödinger-Poisson systems
}

Jing Chen ${ }^{1 *}$ (D) and Ning Zhang ${ }^{2}$

"Correspondence:

cjhnust@aliyun.com

'School of Mathematics and

Computing Sciences, Hunan

University of Science and

Technology, Xiangtan, P.R. China

Full list of author information is

available at the end of the article

\begin{abstract}
This paper is dedicated to studying the following Schrödinger-Poisson system:

$$
\left\{\begin{array}{l}
-\triangle u+V(x) u+K(x) \phi(x) u=f(x, u), \quad x \in \mathbb{R}^{3}, \\
-\triangle \phi=K(x) u^{2}, \quad x \in \mathbb{R}^{3},
\end{array}\right.
$$

where $V(x), K(x)$, and $f(x, u)$ are periodic in $x$. By using the non-Nehari manifold method, we establish the existence of ground state solutions for the above problem under some weak assumptions. Moreover, when $f$ is odd in $u$, we prove that the above problem admits infinitely many geometrically distinct solutions. Our results improve and complement some related literature.
\end{abstract}

MSC: $35 J 10 ; 35 J 20$

Keywords: Schrödinger-Poisson system; Nehari manifold; Ground state; Geometrically distinct solutions

\section{Introduction}

In this paper we are concerned with the nonlinear Schrödinger-Poisson system:

$$
\left\{\begin{array}{l}
-\triangle u+V(x) u+K(x) \phi(x) u=f(x, u), \quad x \in \mathbb{R}^{3}, \\
-\triangle \phi=K(x) u^{2}, \quad x \in \mathbb{R}^{3},
\end{array}\right.
$$

where $V, K: \mathbb{R}^{3} \rightarrow \mathbb{R}$ and $f: \mathbb{R}^{3} \times \mathbb{R} \rightarrow \mathbb{R}$ satisfy the following basic assumptions:

(V0) $V, K \in \mathcal{C}\left(\mathbb{R}^{3},(0, \infty)\right), V(x)$, and $K(x)$ are 1-periodic in $x_{1}, x_{2}$, and $x_{3}$;

(F0) $f(x, t)$ is 1 -periodic in $x_{1}, x_{2}$, and $x_{3}$;

(F1) $f \in \mathcal{C}\left(\mathbb{R}^{3} \times \mathbb{R}, \mathbb{R}\right), f(x, t)=o(|t|)$ uniformly in $x$ as $t \rightarrow 0$, and there exist constants $\mathcal{C}_{0}>0$ and $p \in(2,6)$ such that

$$
|f(x, t)| \leq \mathcal{C}_{0}\left(1+|t|^{p-1}\right), \quad \forall(x, t) \in \mathbb{R}^{3} \times \mathbb{R} .
$$

Schrödinger-Poisson system (also called Schrödinger-Maxwell system) appears in the quantum mechanics model or Hartree-Fock model, which is related to the study of the

(c) The Author(s) 2019. This article is distributed under the terms of the Creative Commons Attribution 4.0 International License (http://creativecommons.org/licenses/by/4.0/), which permits unrestricted use, distribution, and reproduction in any medium, provided you give appropriate credit to the original author(s) and the source, provide a link to the Creative Commons license, and indicate if changes were made. 
interaction of a charged non-relativistic quantum mechanical particle with the electromagnetic field. System (1.1) can be described by coupling a nonlinear Schrödinger and a Poisson equation, from physical point of view, the unknown terms $u$ and $\phi$ are the fields associated to the particle and the electric potential, respectively, the functions $V$ and $K$ are, respectively, an external potential and nonnegative density charge, the nonlinear term $f$ simulates the interaction effect between particles or external nonlinear perturbations, and the coupled term $\phi(x) u$ concerns the interaction with the field. For more details on the physical aspects, we refer the readers to [5-8, 20, 23].

Note that when $\phi \equiv 0,(1.1)$ reduces to the well-known Schrödinger equation, which has been the object of various investigations; see, for example, $[26,33-35,39,40]$ and the references therein.

Under assumption (V0), the set

$$
E=\left\{u \in H^{1}\left(\mathbb{R}^{3}\right): \int_{\mathbb{R}^{3}}\left(|\nabla u|^{2}+V(x) u^{2}\right) \mathrm{d} x<+\infty\right\}
$$

is a Hilbert space equipped with the norm

$$
\|u\|=\left(\int_{\mathbb{R}^{3}}\left(|\nabla u|^{2}+V(x) u^{2}\right) \mathrm{d} x\right)^{1 / 2}
$$

It is well known that the Poisson equation is solved by using the Lax-Milgram theorem. Indeed, as we shall see in Sect. 2, for every $u \in E$, unique $\phi_{u} \in D^{1,2}\left(\mathbb{R}^{3}\right)$ is obtained, such that $-\triangle \phi=K(x) u^{2}$ and so (1.1) can be reduced to a single equation with a nonlocal term

$$
-\triangle u+V(x) u+K(x) \phi_{u}(x) u=f(x, u)
$$

Moreover, (1.2) is variational and its solutions are the critical points of the functional $\Phi$ defined on $E$ by

$$
\Phi(u)=\frac{1}{2} \int_{\mathbb{R}^{3}}\left(|\nabla u|^{2}+V(x) u^{2}\right) \mathrm{d} x+\frac{1}{4} \int_{\mathbb{R}^{3}} K(x) \phi_{u}(x) u^{2} \mathrm{~d} x-\int_{\mathbb{R}^{3}} F(x, u) \mathrm{d} x,
$$

where $F(x, t)=\int_{0}^{t} f(x, s) \mathrm{d} s$. Define

$$
\mathcal{N}:=\left\{u \in E:\left\langle\Phi^{\prime}(u), u\right\rangle=0, u \neq 0\right\}
$$

which is the Nehari manifold of $\Phi$. Let $*$ denote the action of $\mathbb{Z}^{3}$ on $H^{1}\left(\mathbb{R}^{3}\right)$ given by

$$
(k * u)(x)=u(x-k), \quad k \in \mathbb{Z}^{3} .
$$

We note that if $u_{0}$ is a solution of (1.1), then so are $k * u_{0}$ for all $k \in \mathbb{Z}^{3}$. Set

$$
\mathcal{O}(u):=\left\{k * u_{0}, k \in \mathbb{Z}^{3}\right\}
$$

which is called the orbit of $u_{0}$ with respect to the action of $\mathbb{Z}^{3}$. Two solutions $u_{1}$ and $u_{2}$ are said to be geometrically distinct if $\mathcal{O}\left(u_{1}\right)$ and $\mathcal{O}\left(u_{2}\right)$ are disjoint. 
In recent years, there have been rich results for Schrödinger-Poisson systems like (1.1) on the existence of nontrivial solutions, positive solutions, ground states, semi-classical states, and multiple solutions; we refer to $[1-3,6,9,14-17,25,29]$ for the case $f(x, u) \sim$ $|u|^{q-2} u$ with $q \in(4,6) ;[4,28,31,36,41]$ for the case $f(x, u) \sim|u|^{q-2} u$ with $q \in(3,4]$; [13] for the convolution nonlinearity; $[18,38]$ for the critical growth nonlinearity. [19] deals with the multiplicity of solutions for the fractional Schrödinger-Poisson systems. In this paper, we focus on the existence of ground state solutions and infinitely many geometrically distinct solutions for (1.1) in a periodic setting. Let us recall some previous results that led us to the present research.

When the potential and nonlinearity are periodic, that is (V0) and (F0) are satisfied, Zhao and Zhao [41] proved that (1.1) with $K(x)=1$ has a ground solution and infinitely many geometrically distinct solutions by using the Nehari manifold approach, where $f$ and $\partial f / \partial u$ are continuous and satisfy suitable conditions. Based on the generalized Nehari manifold approach developed by Szulkin and Weth [32], Sun and Ma [31] obtained similar results as those in [41], where $f$ satisfies (F0), (F1) and the following assumptions:

(SC) $\lim _{|t| \rightarrow \infty} \frac{F(x, t)}{|t|^{4}}=+\infty$ uniformly in $x \in \mathbb{R}^{3}$;

(MT) $f(x, t) /|t|^{3}$ is increasing in $t$ on $\mathbb{R} \backslash\{0\}$ for every $x \in \mathbb{R}^{3}$.

Later, Chen and Tang relaxed (SC) and (MT) to the following weaker conditions:

(F2) $\lim _{|t| \rightarrow \infty} \frac{F(x, t)}{|t|^{3}}=\infty$ uniformly in $x \in \mathbb{R}^{3}$;

$\left(\mathrm{F}^{\prime}\right)$ there exists $\theta \in(0,1)$ such that

$$
\left[\frac{f(x, \tau)}{\tau^{3}}-\frac{f(x, t \tau)}{(t \tau)^{3}}\right] \operatorname{sign}(1-t)+\theta V(x) \frac{\left|1-t^{2}\right|}{(t \tau)^{2}} \geq 0, \quad \forall x \in \mathbb{R}^{3}, t>0, \tau \neq 0,
$$

and established the existence of ground state solutions for (1.1) by means of the nonNehari manifold method developed by Tang [33-35].

To the best of our knowledge, except for [31, 41], there seems to be no result about the existence of infinitely many geometrically distinct solutions for (1.1). Motivated by the work of $[9,10,31,33]$, in the present paper, we shall establish the existence of ground state solutions and infinitely many geometrically distinct solutions for (1.1) under weaker assumptions than previous works.

Before presenting our theorems, in addition to (V0), (F0), (F1), and (F2), we introduce the following assumptions:

(F3) $\frac{f(x, t)-V(x) t}{|t|^{3}}$ is nondecreasing in $t$ on both $(-\infty, 0)$ and $(0, \infty)$ for every $x \in \mathbb{R}^{3}$;

(F4) there exists a constant $\theta \in[0,1)$ such that

$$
f(x, t) t-4 F(x, t)+\theta V(x) t^{2} \geq 0, \quad \forall(x, t) \in \mathbb{R}^{3} \times \mathbb{R}
$$

(F5) $f(x,-t)=-f(x, t), \forall(x, t) \in \mathbb{R}^{3} \times \mathbb{R}$.

Theorem 1.1 Assume that $V, K$, and $f$ satisfy (V0), (F0), and (F1)-(F3). Then Problem (1.1) has a solution $u_{0} \in E$ such that $\Phi\left(u_{0}\right)=\inf _{\mathcal{N}} \Phi>0$.

Theorem 1.2 Assume that $V, K$, and $f$ satisfy (V0), (F0), and (F1)-(F5). Then Problem (1.1) admits infinitely many pairs of geometrically distinct solutions.

Remark 1.3 Note that the assumptions of Theorems 1.1 and 1.2 are weaker than those of $[10,31,41]$. A simple example of a function satisfying our assumptions but not conditions 
in $[31,41]$ is $f(x, u)=b(x) u^{3}-|u|^{3 / 2} u+|u| u$ for all $(x, u) \in \mathbb{R}^{3} \times \mathbb{R}$, where $b(x)$ is 1-periodic in $x_{1}, x_{2}$, and $x_{3}$ and $\inf _{\mathbb{R}^{3}} b>0$. In this sense, our results improve and complement those of $[10,31,41]$.

To prove Theorem 1.1, following the idea of [10], we apply the non-Nehari manifold method. Unlike the Nehari manifold approach, the key point of this method lies in finding a minimizing Cerami sequence for $\Phi$ outside $\mathcal{N}$ by using a diagonal method. However, the fact that (F3) is weaker than (F3') used in [10] would require our extra efforts. To prove Theorem 1.2, inspired by [31,32], we use deformation type arguments and LusternikSchnirelman theory. However, since $t \mapsto \frac{f(x, t)}{|t|^{3}}$ is not increasing, the generalized Nehari approach developed by [32] does not work. To circumvent this obstacle, we borrow the idea of $[11,12]$ in which Kirchhoff-type problems and Klein-Gordon-Maxwell systems were considered respectively. However, the competing effect of the nonlocal term $\int_{\mathbb{R}^{3}} \phi_{u} u^{2} \mathrm{~d} x$ and the nonlinear term $\int_{\mathbb{R}^{3}} F(u) \mathrm{d} x$ in the expression of $\Phi$ makes our problem more complicated.

The paper is organized as follows. In Sect. 2, we introduce some notation and preliminaries. We complete the proofs of Theorems 1.1 and 1.2 in Sects. 3 and 4, respectively.

Throughout this paper, we denote the norm of $L^{s}\left(\mathbb{R}^{3}\right)$ by $\|u\|_{s}=\left(\int_{\mathbb{R}^{3}}|u|^{s} \mathrm{~d} x\right)^{1 / s}$ for $s \geq 2$, $B_{r}(x)=\left\{y \in \mathbb{R}^{3}:|y-x|<r\right\}$, and positive constants possibly different in different places by $C_{1}, C_{2}, \ldots$

\section{Notation and preliminaries}

Hereafter, $H^{1}\left(\mathbb{R}^{3}\right)$ is the usual Sobolev space with the standard scalar product and norm

$$
(u, v)_{H^{1}}=\int_{\mathbb{R}^{3}}(\nabla u \nabla v+u v) \mathrm{d} x, \quad\|u\|_{H^{1}}^{2}=\int_{\mathbb{R}^{3}}\left(|\nabla u|^{2}+u^{2}\right) \mathrm{d} x,
$$

and

$$
D^{1,2}\left(\mathbb{R}^{3}\right)=\left\{u \in L^{6}\left(\mathbb{R}^{3}\right): \nabla u \in L^{2}\left(\mathbb{R}^{3}\right)\right\}
$$

equipped with the norm defined by

$$
\|u\|_{D^{1,2}}^{2}=\int_{\mathbb{R}^{3}}|\nabla u|^{2} \mathrm{~d} x
$$

It is easy to show that (1.1) can be reduced to a single equation with a nonlocal term. Namely, for any $K u^{2} \in L_{\text {loc }}^{1}\left(\mathbb{R}^{3}\right)$ such that

$$
\int_{\mathbb{R}^{3}} \int_{\mathbb{R}^{3}} \frac{u^{2}(x) u^{2}(y)}{|x-y|} \mathrm{d} x \mathrm{~d} y<\infty,
$$

the distributional solution

$$
\phi_{u}(x)=\int_{\mathbb{R}^{3}} \frac{K(y) u^{2}(y)}{|x-y|} \mathrm{d} y=\frac{1}{|x|} * K u^{2}
$$

of the Poisson equation

$$
-\triangle \phi=K(x) u^{2}, \quad x \in \mathbb{R}^{3}
$$


belongs to $D^{1,2}\left(\mathbb{R}^{3}\right)$ and is the unique weak solution in $D^{1,2}\left(\mathbb{R}^{3}\right)$ (see, e.g., [29] for more details), and

$$
\begin{aligned}
& \int_{\mathbb{R}^{3}} \nabla \phi_{u} \nabla v \mathrm{~d} x=\int_{\mathbb{R}^{3}} K(x) u^{2} v \mathrm{~d} x, \quad \forall v \in H^{1}\left(\mathbb{R}^{3}\right), \\
& \int_{\mathbb{R}^{3}} \int_{\mathbb{R}^{3}} \frac{K(x) K(y)}{|x-y|} u^{2}(x) u^{2}(y) \mathrm{d} x \mathrm{~d} y=\int_{\mathbb{R}^{3}} K(x) \phi_{u}(x) u^{2} \mathrm{~d} x .
\end{aligned}
$$

Moreover, $\phi_{u}(x)>0$ when $u \neq 0$, because $K$ does (see (V0)). By using Hardy-LittlewoodSobolev inequality (see [21] or [22, p. 98]), we have the following inequality:

$$
\int_{\mathbb{R}^{3}} \int_{\mathbb{R}^{3}} \frac{|u(x) v(y)|}{|x-y|} \mathrm{d} x \mathrm{~d} y \leq \frac{8 \sqrt[3]{2}}{3 \sqrt[3]{\pi}}\|u\|_{6 / 5}\|v\|_{6 / 5}, \quad u, v \in L^{6 / 5}\left(\mathbb{R}^{3}\right) .
$$

Formally, the solutions of (1.1) are then the critical points of the reduced functional (1.3). Indeed, (V0), (F0), and (2.4) imply that $\Phi$ is a well-defined functional of class $\mathcal{C}^{1}$ and that

$$
\left\langle\Phi^{\prime}(u), v\right\rangle=\int_{\mathbb{R}^{3}}(\nabla u \nabla v+V(x) u v) \mathrm{d} x+\int_{\mathbb{R}^{3}}\left[K(x) \phi_{u}(x) u-f(x, u)\right] v \mathrm{~d} x .
$$

Hence if $u \in E$ is a critical point of $\Phi$, then the pair $\left(u, \phi_{u}\right)$, with $\phi_{u}$ as in (2.1), is a solution of (1.1).

Lemma 2.1 Under assumptions (V0), (F1), and (F2),

$$
\Phi(u) \geq \Phi(t u)+\frac{1-t^{4}}{4}\left\langle\Phi^{\prime}(u), u\right\rangle+\frac{\left(1-t^{2}\right)^{2}}{4}\|\nabla u\|_{2}^{2}, \quad \forall u \in E, t \geq 0 .
$$

Proof For any $x \in \mathbb{R}^{3}, t \geq 0, \tau \neq 0$, (F2) yields

$$
\begin{aligned}
& \frac{1-t^{4}}{4} \tau f(x, \tau)+F(x, t \tau)-F(x, \tau)+\frac{V(x)}{4}\left(1-t^{2}\right)^{2} \tau^{2} \\
& =\int_{t}^{1}\left[\frac{f(x, \tau)}{\tau^{3}}-\frac{f(x, s \tau)}{(s \tau)^{3}}+V(x) \frac{\left(1-s^{2}\right)}{(s \tau)^{2}}\right] s^{3} \tau^{4} \mathrm{~d} s \geq 0 .
\end{aligned}
$$

Note that

$$
\Phi(u)=\frac{1}{2}\|u\|^{2}+\frac{1}{4} \int_{\mathbb{R}^{3}} K(x) \phi_{u}(x) u^{2} \mathrm{~d} x-\int_{\mathbb{R}^{3}} F(x, u) \mathrm{d} x
$$

and

$$
\left\langle\Phi^{\prime}(u), u\right\rangle=\|u\|^{2}+\int_{\mathbb{R}^{3}} K(x) \phi_{u}(x) u^{2} \mathrm{~d} x-\int_{\mathbb{R}^{3}} f(x, u) u \mathrm{~d} x .
$$

Thus, by (2.7), (2.8), and (2.9), one has

$$
\begin{aligned}
\Phi(u)-\Phi(t u) & =\frac{1-t^{2}}{2}\|u\|^{2}+\frac{1-t^{4}}{4} \int_{\mathbb{R}^{3}} K(x) \phi_{u}(x) u^{2} \mathrm{~d} x+\int_{\mathbb{R}^{3}}[F(x, t u)-F(x, u)] \mathrm{d} x \\
& =\frac{1-t^{4}}{4}\left\langle\Phi^{\prime}(u), u\right\rangle+\frac{\left(1-t^{2}\right)^{2}}{4}\|\nabla u\|_{2}^{2}
\end{aligned}
$$




$$
\begin{aligned}
& +\int_{\mathbb{R}^{3}}\left[\frac{1-t^{4}}{4} f(x, u) u+F(x, t u)-F(x, u)+\frac{V(x)}{4}\left(1-t^{2}\right)^{2} u^{2}\right] \mathrm{d} x \\
\geq & \frac{1-t^{4}}{4}\left\langle\Phi^{\prime}(u), u\right\rangle+\frac{\left(1-t^{2}\right)^{2}}{4}\|\nabla u\|_{2}^{2}, \quad t \geq 0 .
\end{aligned}
$$

This shows that (2.6) holds.

Corollary 2.2 Under assumptions (V0), (F1), and (F2), for $u \in \mathcal{N}$,

$$
\Phi(u)=\max _{t \geq 0} \Phi(t u)
$$

Unlike the super-cubic case, to show $\mathcal{N} \neq \emptyset$ in our situation, we have to overcome the competing effect of the nonlocal term. Inspired by Chen and Tang [10], we define a set $\Lambda$ as follows:

$$
\Lambda=\left\{u \in E: \int_{\mathbb{R}^{3}}\left[V(x) u^{2}+K(x) \phi_{u} u^{2}-f(x, u) u\right] \mathrm{d} x<0\right\} .
$$

Lemma 2.3 Under assumptions (V0) and (F1)-(F3), $\Lambda \neq \emptyset$ and $\mathcal{N} \subset \Lambda$. Then, for any $u \in \Lambda$, there exists unique $t(u)>0$ such that $t(u) u \in \mathcal{N}$.

Proof First, we show that $\Lambda \neq \emptyset$. From (2.4) and Sobolev imbedding theorem, there exists $C_{1}>0$ such that $\int_{\mathbb{R}^{3}} \phi_{u} u^{2} \mathrm{~d} x \leq C_{1}\|u\|^{4}$ for all $u \in E$. For any fixed $u \in E$ with $u \neq 0$, set $u_{t}(x)=u(t x)$ for $t>0$. By (V0), one has

$$
\begin{aligned}
& \int_{\mathbb{R}^{3}}\left[V(x)\left(t u_{t}\right)^{2}+K(x) \phi_{\left(t u_{t}\right)}\left(t u_{t}\right)^{2}-f\left(x, t u_{t}\right) t u_{t}\right] \mathrm{d} x \\
& \quad=t^{-1} \int_{\mathbb{R}^{3}} V\left(t^{-1} x\right) u^{2} \mathrm{~d} x+t^{-1} \int_{\mathbb{R}^{3}} K\left(t^{-1} x\right) \phi_{u} u^{2} \mathrm{~d} x-\int_{\mathbb{R}^{3}} \frac{f\left(t^{-1} x, t u\right) t u}{t^{3}} \mathrm{~d} x \\
& \quad \leq V_{\infty} t^{-1}\|u\|_{2}^{2}+C_{1} K_{\infty} t^{-1}\|u\|^{4}-\int_{\mathbb{R}^{3}} \frac{f\left(t^{-1} x, t u\right) t u}{t^{3}} \mathrm{~d} x
\end{aligned}
$$

where $V_{\infty}=\sup _{x \in \mathbb{R}^{3}} V(x)$ and $K_{\infty}=\sup _{x \in \mathbb{R}^{3}} K(x)$. Note that, for $u(x) \neq 0, F\left(t^{-1} x, t u\right) /$ $|t u|^{3} \rightarrow+\infty$ as $t \rightarrow+\infty$ uniformly in $x \in \mathbb{R}^{3}$ by (F3), and (2.7) with $t=0$ yields

$$
\frac{1}{4} f(x, \tau) \tau-F(x, \tau)+\frac{V(x)}{4} \tau^{2} \geq 0, \quad \forall x \in \mathbb{R}^{3}, \tau \in \mathbb{R},
$$

then we have

$$
\frac{f\left(t^{-1} x, t u\right) t u}{|t u|^{3}} \rightarrow+\infty, \quad \text { as } t \rightarrow+\infty \text { uniformly in } x \in \mathbb{R}^{3} .
$$

Thus, it follows from (V0), (2.11), and (2.13) that

$$
\int_{\mathbb{R}^{3}}\left[V(x)\left(t u_{t}\right)^{2}+K(x) \phi_{\left(t u_{t}\right)}\left(t u_{t}\right)^{2}-f\left(x, t u_{t}\right) t u_{t}\right] \mathrm{d} x \rightarrow-\infty, \quad \text { as } t \rightarrow+\infty .
$$

Thus, taking $v=T u_{T}$ for $T$ large, we have $v \in \Lambda$. Hence, $\Lambda \neq \emptyset$. From (2.5), it is easy to see that $\mathcal{N} \subset \Lambda$. 
Next, we prove the last part of the lemma. Let $u \in \Lambda$ be fixed and define a function $g(t):=\left\langle\Phi^{\prime}(t u), t u\right\rangle$ on $[0, \infty)$. By (F2), one has

$$
f(x, t \tau) t \tau \geq f(x, \tau) \tau t^{4}-V(x)\left(t^{2}-1\right)(t \tau)^{2}, \quad \forall x \in \mathbb{R}^{3}, t \geq 1, \tau \in \mathbb{R},
$$

which yields

$$
\begin{aligned}
& \int_{\mathbb{R}^{3}}\left[V(x)(t \tau)^{2}+K(x) \phi_{t \tau}(t \tau)^{2}-f(x, t \tau) t \tau\right] \mathrm{d} x \\
& \quad \leq t^{4} \int_{\mathbb{R}^{3}}\left[V(x) \tau^{2}+K(x) \phi_{\tau} \tau^{2}-f(x, \tau) \tau\right] \mathrm{d} x, \quad \forall t \geq 1, \tau \in \mathbb{R} .
\end{aligned}
$$

From (2.5) and (2.15) it follows that

$$
\begin{aligned}
g(t) \leq & t^{2}\|u\|^{2}+t^{4} \int_{\mathbb{R}^{3}}\left[V(x) u^{2}+K(x) \phi_{u} u^{2}-f(x, u) u\right] \mathrm{d} x \\
& -t^{2} \int_{\mathbb{R}^{3}} V(x) u^{2} \mathrm{~d} x, \quad \forall t \geq 1 .
\end{aligned}
$$

Using (F0), (2.5), and (2.16), it is easy to verify that $g(0)=0, g(t)>0$ for $t>0$ small and $g(t)<0$ for $t$ large due to $u \in \Lambda$. Therefore, there exists $t_{0}=t(u)>0$ so that $g\left(t_{0}\right)=0$ and $t(u) u \in \mathcal{N}$. We claim that $t(u)$ is unique for any $u \in \Lambda$. In fact, for any given $u \in \Lambda$, let $t_{1}, t_{2}>0$ such that $g\left(t_{1}\right)=g\left(t_{2}\right)=0$. Jointly with (2.6), we have

$$
\begin{aligned}
\Phi\left(t_{1} u\right) & \geq \Phi\left(t_{2} u\right)+\frac{t_{1}^{4}-t_{2}^{4}}{4 t_{1}^{4}}\left\langle\Phi^{\prime}\left(t_{1} u\right), t_{1} u\right\rangle+\frac{\left(t_{1}^{2}-t_{2}^{2}\right)^{2}}{4 t_{1}^{2}}\|\nabla u\|_{2}^{2} \\
& =\Phi\left(t_{2} u\right)+\frac{\left(t_{1}^{2}-t_{2}^{2}\right)^{2}}{4 t_{1}^{2}}\|\nabla u\|_{2}^{2}
\end{aligned}
$$

and

$$
\begin{aligned}
\Phi\left(t_{2} u\right) & \geq \Phi\left(t_{1} u\right)+\frac{t_{2}^{4}-t_{1}^{4}}{4 t_{2}^{4}}\left\langle\Phi^{\prime}\left(t_{2} u\right), t_{2} u\right\rangle+\frac{\left(t_{2}^{2}-t_{1}^{2}\right)^{2}}{4 t_{2}^{2}}\|\nabla u\|_{2}^{2} \\
& =\Phi\left(t_{1} u\right)+\frac{\left(t_{2}^{2}-t_{1}^{2}\right)^{2}}{4 t_{2}^{2}}\|\nabla u\|_{2}^{2} .
\end{aligned}
$$

(2.17) and (2.18) imply $t_{1}=t_{2}$. Hence, $t(u)>0$ is unique for any $u \in \Lambda$.

Lemma 2.4 Under assumptions (V0) and (F1)-(F3), then

$$
\inf _{u \in \mathcal{N}} \Phi(u):=c=\inf _{u \in \Lambda, u \neq 0} \max _{t \geq 0} \Phi(t u)>0 .
$$

Proof Both Corollary 2.2 and Lemma 2.3 imply that $c=\inf _{u \in \Lambda, u \neq 0} \max _{t \geq 0} \Phi(t u)$. Using Lemma 2.1, it is easy to see that $c>0$.

Lemma 2.5 Under assumptions (V0) and (F1)-(F3), there exist a constant $c_{*} \in(0, c]$ and a sequence $\left\{u_{n}\right\} \subset E$ satisfying

$$
\Phi\left(u_{n}\right) \rightarrow c_{*}, \quad\left\|\Phi^{\prime}\left(u_{n}\right)\right\|\left(1+\left\|u_{n}\right\|\right) \rightarrow 0 .
$$


Proof By (F1) and (1.3), we know that there exist $\delta_{0}>0$ and $\rho_{0}>0$ such that

$$
\Phi(u) \geq \rho_{0}, \quad\|u\|=\delta_{0} .
$$

In view of Lemmas 2.3 and 2.4, we may choose $v_{k} \in \mathcal{N} \subset \Lambda$ such that

$$
c-\frac{1}{k}<\Phi\left(v_{k}\right)<c+\frac{1}{k}, \quad k \in \mathbb{N} .
$$

Using Lemma 2.1 and (2.20), it is easy to check that $\Phi\left(t v_{k}\right) \geq \rho_{0}$ for small $t>0$ and $\Phi\left(t v_{k}\right)<$ 0 for large $t>0$ due to $v_{k} \in \Lambda$. Since $\Phi(0)=0$, then the mountain pass lemma implies that there exists a sequence $\left\{u_{k, n}\right\}_{n \in \mathbb{N}} \subset E$ satisfying

$$
\Phi\left(u_{k, n}\right) \rightarrow c_{k}, \quad\left\|\Phi^{\prime}\left(u_{k, n}\right)\right\|\left(1+\left\|u_{k, n}\right\|\right) \rightarrow 0, \quad k \in \mathbb{N},
$$

where $c_{k} \in\left[\rho_{0}, \sup _{t \geq 0} \Phi\left(t v_{k}\right)\right]$. By virtue of Corollary 2.2, one has $\Phi\left(v_{k}\right)=\sup _{t \geq 0} \Phi\left(t v_{k}\right)$. Hence, by (2.21) and (2.22), one has

$$
\Phi\left(u_{k, n}\right) \rightarrow c_{k} \in\left[\rho_{0}, c+\frac{1}{k}\right), \quad\left\|\Phi^{\prime}\left(u_{k, n}\right)\right\|\left(1+\left\|u_{k, n}\right\|\right) \rightarrow 0, \quad k \in \mathbb{N}
$$

Now, we can choose a sequence $\left\{n_{k}\right\} \subset \mathbb{N}$ such that

$$
\Phi\left(u_{k, n_{k}}\right) \in\left[\rho_{0}, c+\frac{1}{k}\right), \quad\left\|\Phi^{\prime}\left(u_{k, n_{k}}\right)\right\|\left(1+\left\|u_{k, n_{k}}\right\|\right)<\frac{1}{k}, \quad k \in \mathbb{N} .
$$

Let $u_{k}=u_{k, n_{k}}, k \in \mathbb{N}$. Then, going if necessary to a subsequence, we have

$$
\Phi\left(u_{n}\right) \rightarrow c_{*} \in\left[\rho_{0}, c\right], \quad\left\|\Phi^{\prime}\left(u_{n}\right)\right\|\left(1+\left\|u_{n}\right\|\right) \rightarrow 0 .
$$

Lemma 2.6 Under assumptions (V0) and (F1)-(F3), any sequence $\left\{u_{n}\right\} \subset E$ satisfying (2.19) is bounded in $E$.

Proof By (2.6) with $t=0$, one has

$$
c_{*}+o(1)=\Phi\left(u_{n}\right)-\frac{1}{4}\left\langle\Phi^{\prime}\left(u_{n}\right), u_{n}\right\rangle \geq \frac{1}{4}\left\|\nabla u_{n}\right\|_{2}^{2} .
$$

By (V0), it is easy to see that there exists $V_{0}>0$ such that $V(x) \geq V_{0}$ for all $x \in \mathbb{R}^{3}$. Since $\left\langle\Phi^{\prime}\left(u_{n}\right), u_{n}\right\rangle=o(1)$, it follows from (V0), (F1), and the Sobolev embedding inequality that

$$
\begin{aligned}
V_{0}\left\|u_{n}\right\|_{2}^{2} & \leq\left\|u_{n}\right\|^{2}+\int_{\mathbb{R}^{3}} K(x) \phi_{u_{n}} u_{n}^{2} \mathrm{~d} x \\
& =\int_{\mathbb{R}^{3}} f\left(u_{n}\right) u_{n} \mathrm{~d} x+o(1) \\
& \leq \frac{1}{2} V_{0}\left\|u_{n}\right\|_{2}^{2}+C_{2}\left\|u_{n}\right\|_{6}^{6}+o(1) \\
& \leq \frac{1}{2} V_{0}\left\|u_{n}\right\|_{2}^{2}+C_{2} S^{-3}\left\|\nabla u_{n}\right\|_{2}^{6}+o(1),
\end{aligned}
$$

which together with (2.25) implies that $\left\{u_{n}\right\}$ is bounded in $E$. 


\section{Ground state solutions}

In this section, we give the proof of Theorem 1.1.

Proof of Theorem 1.1 Lemma 2.5 implies the existence of a sequence $\left\{u_{n}\right\} \subset E$ satisfying (2.19), then

$$
\Phi\left(u_{n}\right) \rightarrow c_{*}>0, \quad\left\langle\Phi^{\prime}\left(u_{n}\right), u_{n}\right\rangle \rightarrow 0 .
$$

By Lemma 2.6, $\left\{u_{n}\right\}$ is bounded in $E$. If

$$
\delta:=\limsup _{n \rightarrow \infty} \sup _{y \in \mathbb{R}^{3}} \int_{B_{1}(y)}\left|u_{n}\right|^{2} \mathrm{~d} x=0
$$

then by Lion's concentration compactness principle [24] or [37, Lemma 1.21], $u_{n} \rightarrow 0$ in $L^{s}\left(\mathbb{R}^{3}\right)$ for $2<s<6$. Moreover, there exists $C_{3}>0$ such that $\left\|u_{n}\right\|_{2} \leq C_{3}$. By (F0), for $\varepsilon=c_{*} / 2 C_{3}^{2}$, there exists $C_{\varepsilon}>0$ such that

$$
\limsup _{n \rightarrow \infty} \int_{\mathbb{R}^{3}}\left|\frac{1}{2} f\left(x, u_{n}\right) u_{n}-F\left(x, u_{n}\right)\right| \mathrm{d} x \leq \frac{3}{2} \varepsilon C_{3}^{2}+C_{\varepsilon} \lim _{n \rightarrow \infty}\left\|u_{n}\right\|_{p}^{p}=\frac{3 c_{*}}{4} .
$$

By (V0), (2.3), and (2.4), we have

$$
\begin{aligned}
\limsup _{n \rightarrow \infty} \int_{\mathbb{R}^{3}} K(x) \phi_{u_{n}}(x) u_{n}^{2} \mathrm{~d} x & =\limsup _{n \rightarrow \infty} \int_{\mathbb{R}^{3}} \int_{\mathbb{R}^{3}} \frac{K(x) K(y)}{|x-y|} u_{n}^{2}(x) u_{n}^{2}(y) \mathrm{d} x \mathrm{~d} y \\
& \leq K_{\infty}^{2} \limsup _{n \rightarrow \infty} \int_{\mathbb{R}^{3}} \int_{\mathbb{R}^{3}} \frac{u_{n}^{2}(x) u_{n}^{2}(y)}{|x-y|} \mathrm{d} x \mathrm{~d} y \\
& \leq \mathcal{C}_{1} K_{\infty}^{2} \limsup _{n \rightarrow \infty}\left\|u_{n}\right\|_{12 / 5}^{4}=0,
\end{aligned}
$$

where, and in the sequel, $\mathcal{C}_{1}=8 \sqrt[3]{2} / 3 \sqrt[3]{\pi}$. From (1.3), (2.5), (3.1), (3.2), and (3.3), one has

$$
\begin{aligned}
c_{*} & =\Phi\left(u_{n}\right)-\frac{1}{2}\left\langle\Phi^{\prime}\left(u_{n}\right), u_{n}\right\rangle+o(1) \\
& =-\frac{1}{4} \int_{\mathbb{R}^{3}} K(x) \phi_{u_{n}}(x) u_{n}^{2} \mathrm{~d} x+\int_{\mathbb{R}^{3}}\left[\frac{1}{2} f\left(x, u_{n}\right) u_{n}-F\left(x, u_{n}\right)\right] \mathrm{d} x+o(1) \\
& \leq \frac{3 c_{*}}{4}+o(1) .
\end{aligned}
$$

This contradiction shows $\delta>0$.

Going if necessary to a subsequence, we may assume the existence of $k_{n} \in \mathbb{Z}^{3}$ such that

$$
\int_{B_{2}\left(k_{n}\right)}\left|u_{n}\right|^{2} \mathrm{~d} x>\frac{\delta}{2} .
$$

Let $v_{n}(x)=u_{n}\left(x+k_{n}\right)$. Then

$$
\int_{B_{2}(0)}\left|v_{n}\right|^{2} \mathrm{~d} x>\frac{\delta}{2} .
$$


Since $V(x), K(x)$, and $f(x, u)$ are periodic on $x$, we have

$$
\Phi\left(v_{n}\right) \rightarrow c_{*} \in(0, c], \quad\left\|\Phi^{\prime}\left(v_{n}\right)\right\|\left(1+\left\|v_{n}\right\|\right) \rightarrow 0 .
$$

Passing to a subsequence, we have $v_{n} \rightarrow \bar{v}$ in $E, v_{n} \rightarrow \bar{v}$ in $L_{\text {loc }}^{s}\left(\mathbb{R}^{3}\right), 2 \leq s<6$, and $v_{n}(x) \rightarrow$ $\bar{v}(x)$ a.e. on $\mathbb{R}^{3}$. Thus, (3.5) implies that $\bar{v} \neq 0$. For every $\phi \in C_{0}^{\infty}\left(\mathbb{R}^{3}\right)$, we have

$$
\left\langle\Phi^{\prime}(\bar{v}), \phi\right\rangle=\lim _{n \rightarrow \infty}\left\langle\Phi^{\prime}\left(v_{n}\right), \phi\right\rangle=0
$$

Hence $\Phi^{\prime}(\bar{v})=0$. This shows that $\bar{v} \in \mathcal{N}$ is a nontrivial solution of Problem (1.1) and $\Phi(\bar{v}) \geq c$. It follows from (F2), (3.6), and Fatou's lemma that

$$
\begin{aligned}
c & \geq c_{*}=\lim _{n \rightarrow \infty}\left[\Phi\left(v_{n}\right)-\frac{1}{4}\left\langle\Phi^{\prime}\left(v_{n}\right), v_{n}\right\rangle\right] \\
& =\lim _{n \rightarrow \infty}\left\{\frac{1}{4}\left\|\nabla v_{n}\right\|_{2}^{2}+\int_{\mathbb{R}^{3}}\left[\frac{1}{4} f\left(x, v_{n}\right) v_{n}-F\left(x, v_{n}\right)+\frac{V(x)}{4} v_{n}^{2}\right] \mathrm{d} x\right\} \\
& \geq \frac{1}{4} \liminf _{n \rightarrow \infty}\left\|\nabla v_{n}\right\|_{2}^{2}+\liminf _{n \rightarrow \infty} \int_{\mathbb{R}^{3}}\left[\frac{1}{4} f\left(x, v_{n}\right) v_{n}-F\left(x, v_{n}\right)+\frac{V(x)}{4} v_{n}^{2}\right] \mathrm{d} x \\
& \geq \frac{1}{4}\|\bar{v}\|^{2}+\int_{\mathbb{R}^{3}}\left[\frac{1}{4} f(x, \bar{v}) \bar{v}-F(x, \bar{v})\right] \mathrm{d} x \\
& =\Phi(\bar{v})-\frac{1}{4}\left\langle\Phi^{\prime}(\bar{v}), \bar{v}\right\rangle=\Phi(\bar{v}) .
\end{aligned}
$$

This shows that $\Phi(\bar{v}) \leq c$, and so $\Phi(\bar{v})=c=\inf _{\mathcal{N}} \Phi>0$.

\section{Infinitely many geometrically distinct solutions}

To prove Theorem 1.2, we need some notations. For $d_{2} \geq d_{1}>-\infty$ and $c \in \mathbb{R}$, we put

$$
\begin{aligned}
& \Phi^{d_{2}}:=\left\{u \in E: \Phi(u) \leq d_{2}\right\}, \quad \Phi_{d_{1}}:=\left\{u \in E: \Phi(u) \geq d_{1}\right\}, \quad \Phi_{d_{1}}^{d_{2}}:=\Phi_{d_{1}} \cap \Phi^{d_{2}} ; \\
& \mathcal{K}:=\left\{u \in E \backslash\{0\}: \Phi^{\prime}(u)=0\right\}, \quad \mathcal{K}_{c}:=\{u \in \mathcal{K}: \Phi(u)=c\} .
\end{aligned}
$$

In view of Theorem 1.1, under (V0), (F0), and (F1)-(F3), (1.1) has a nontrivial solution $\bar{u} \in H^{1}\left(\mathbb{R}^{N}\right)$ satisfying $\Phi(\bar{u})=c_{0}:=\inf _{\mathcal{K}} \Phi>0$. Therefore, $\mathcal{K} \supseteq \mathcal{K}_{c_{0}} \neq \emptyset$. Following the strategy of [32], we choose a subset $\mathcal{F}$ of $\mathcal{K}$ such that $\mathcal{F}=-\mathcal{F}$ and each orbit $\mathcal{O}(w) \subset \mathcal{K}$ has a unique representative in $\mathcal{F}$. It suffices to show that the set $\mathcal{F}$ is infinite. So from now on we assume by contradiction that

$\mathcal{F}$ is a finite set.

Lemma 4.1 $\kappa:=\inf \{\|u-v\|: u, v \in \mathcal{K}, u \neq v\}>0$.

Proof Choose $\left\{u_{n}\right\},\left\{v_{n}\right\} \subset \mathcal{K}$ such that $\left\|u_{n}-v_{n}\right\| \rightarrow \kappa$. Then there exist $w^{1}, w^{2} \in \mathcal{F}$ and $k_{n}, l_{n} \in \mathbb{Z}^{3}$ such that $u_{n}=w^{1}\left(\cdot-k_{n}\right)$ and $v_{n}=w^{2}\left(\cdot-l_{n}\right)$. Put $m_{n}=k_{n}-l_{n}$. There are two possible cases.

Case (1). $\left\{\left|m_{n}\right|\right\}$ is bounded. Passing to a subsequence, $m_{n}=m \in \mathbb{Z}^{3}$, one has

$$
\kappa+o(1)=\left\|u_{n}-v_{n}\right\|=\left\|w^{1}\left(\cdot-k_{n}\right)-w^{2}\left(\cdot-l_{n}\right)\right\|=\left\|w^{1}-w^{2}(\cdot-m)\right\|>0 .
$$


Case (2). $\left\{\left|m_{n}\right|\right\}$ is unbounded. Passing to a subsequence, $\left|m_{n}\right| \rightarrow \infty$, one has

$$
\begin{aligned}
\kappa+o(1) & =\left\|u_{n}-v_{n}\right\|=\left\|w^{1}\left(\cdot-k_{n}\right)-w^{2}\left(\cdot-l_{n}\right)\right\| \\
& =\left\|w^{1}-w^{2}\left(\cdot-m_{n}\right)\right\|=\left(\left\|w^{1}\right\|^{2}+\left\|w^{2}\right\|^{2}\right)^{1 / 2}+o(1)>0 .
\end{aligned}
$$

Both Cases (1) and (2) show that $\kappa>0$.

Lemma 4.2 (Discreteness of (PS)-sequences) Let $c \geq c_{0}$. If $\left\{u_{n}^{1}\right\},\left\{u_{n}^{2}\right\} \subset \Phi^{c}$ are two (PS)-sequences for $\Phi$, then either $\left\|u_{n}^{1}-u_{n}^{2}\right\| \rightarrow 0$ as $n \rightarrow \infty$ or $\liminf _{n \rightarrow \infty}\left\|u_{n}^{1}-u_{n}^{2}\right\| \geq$ $\min \left\{\kappa, \sqrt{2} c_{0}\right\}>0$.

Proof First, we prove the boundedness of Palais-Smale sequences for $\Phi$. Let $\left\{u_{n}\right\}$ be such that

$$
\Phi\left(u_{n}\right) \rightarrow c>0, \quad \Phi^{\prime}\left(u_{n}\right) \rightarrow 0 .
$$

Then it follows from (F4) and (4.2) that, for large $n \in \mathbb{N}$,

$$
\begin{aligned}
c+1+\left\|u_{n}\right\| & \geq \Phi\left(u_{n}\right)-\frac{1}{4}\left\langle\Phi^{\prime}\left(u_{n}\right), u_{n}\right\rangle \\
& =\frac{1}{4}\left\|u_{n}\right\|^{2}+\int_{\mathbb{R}^{3}}\left[\frac{1}{4} f\left(x, u_{n}\right) u_{n}-F\left(x, u_{n}\right)\right] \mathrm{d} x \\
& \geq \frac{1-\theta}{4}\left\|u_{n}\right\|^{2},
\end{aligned}
$$

which implies that $\left\{u_{n}\right\}$ is bounded in $E$. Thus, $\left\{u_{n}^{1}\right\},\left\{u_{n}^{2}\right\} \subset \Phi^{c}$ are two bounded (PS)sequences for $\Phi$. Next, we fix $p$ as in (F1), and we distinguish two cases.

Case (1). $\left\|u_{n}^{1}-u_{n}^{2}\right\|_{p} \rightarrow 0$. In this case, we can prove that $\lim _{n \rightarrow \infty}\left\|u_{n}^{1}-u_{n}^{2}\right\|=0$ in a similar fashion as [32, Lemma 2.14].

Case (2). $\left\|u_{n}^{1}-u_{n}^{2}\right\|_{p} \nrightarrow 0$. Then again by [37, Lemma 1.21], there exist $\varepsilon_{0}>0$ and $k_{n} \in \mathbb{Z}^{3}$ such that, after passing to a subsequence,

$$
\int_{B_{1+\sqrt{N}}\left(k_{n}\right)}\left|u_{n}^{1}-u_{n}^{2}\right|^{p} \mathrm{~d} x=\max _{k \in \mathbb{Z}^{3}} \int_{B_{1+\sqrt{N}}(k)}\left|u_{n}^{1}-u_{n}^{2}\right|^{p} \mathrm{~d} x \geq \varepsilon_{0} .
$$

Using that $\Phi$ is equivariant with respect to translations of the form $u \mapsto u(\cdot-k)$ with $k \in \mathbb{Z}^{3}$, we may assume that $\left\{k_{n}\right\}$ is bounded in $\mathbb{Z}^{3}$. We may pass to a subsequence such that

$$
u_{n}^{1} \rightarrow u^{1}, \quad u_{n}^{2} \rightarrow u^{2}, \quad u^{1} \neq u^{2}, \quad \Phi^{\prime}\left(u^{1}\right)=\Phi^{\prime}\left(u^{2}\right)=0 .
$$

We first consider the case where $u^{1} \neq 0$ and $u^{2} \neq 0$, so that $u^{1}, u^{2} \in \mathcal{K}$. By Lemma 4.1 , one has $\liminf _{n \rightarrow \infty}\left\|u_{n}^{1}-u_{n}^{2}\right\| \geq\left\|u^{1}-u^{2}\right\| \geq \kappa$. It remains to consider the case where either $u^{2}=0$ or $u^{1}=0$. In this case, it is easy to see that $\liminf _{n \rightarrow \infty}\left\|u_{n}^{1}-u_{n}^{2}\right\| \geq\left\|u^{1}-u^{2}\right\| \geq$ $\sqrt{2} c_{0}$.

Since $\Phi$ is even, it is known (see, e.g., [30, Lemma II.3.9]) that $\Phi$ admits an odd pseudogradient vector field, i.e., there exists an odd Lipschitz continuous map $W: E \backslash(\mathcal{K} \cup\{0\}) \rightarrow$ 
E such that

$$
\left.\begin{array}{l}
\|W(u)\| \leq 2\left\|\Phi^{\prime}(u)\right\|, \\
\left\langle\Phi^{\prime}(u), W(u)\right\rangle \geq\left\|\Phi^{\prime}(u)\right\|^{2} .
\end{array}\right\}
$$

Now we consider the Cauchy problem:

$$
\left\{\begin{array}{l}
\frac{d \eta}{d t}=-W(\eta) \\
\eta(0, u)=u
\end{array}\right.
$$

The basic existence-uniqueness theorem for ordinary differential equations implies that, for each $u \in E$, (4.4) has a unique solution $\eta(t, u)$ defined for $t$ in a maximal interval $\left(T^{-}(u), T^{+}(u)\right)$, and $\eta(t, u)$ is odd with respect to $u \in E$.

Lemma 4.3 Let $u \in E \backslash(\mathcal{K} \cup\{0\})$. If $\inf _{t \in\left[0, T^{+}(u)\right)} \Phi(\eta(t, u))>-\infty$, then $\lim _{t \rightarrow T^{+}(u)} \eta(t, u)$ exists and is a critical point of $\Phi$.

Proof From (4.3) and (4.4), we have

$$
\begin{aligned}
\frac{d}{d t} \Phi(\eta(t, u)) & =-\left\langle\Phi^{\prime}(\eta(t, u)), W(\eta(t, u))\right\rangle \\
& \leq-\left\|\Phi^{\prime}(\eta(t, u))\right\|^{2}<0, \quad \forall t \in\left[0, T^{+}(u)\right) .
\end{aligned}
$$

This shows that $\Phi(\eta(t, u))$ is strictly decreasing on $t \in\left[0, T^{+}(u)\right)$, and so $\tau:=$ $\lim _{t \rightarrow T^{+}(u)} \Phi(\eta(t, u))$ exists.

Case (1). $T^{+}(u)<+\infty$. For $0 \leq t_{1}<t_{2}<T^{+}(u)$, from (4.3) and (4.4), we have

$$
\begin{aligned}
\left\|\eta\left(t_{2}, u\right)-\eta\left(t_{1}, u\right)\right\| & \leq \int_{t_{1}}^{t_{2}}\|W(\eta(t, u))\| \mathrm{d} t \\
& \leq 2 \int_{t_{1}}^{t_{2}} \sqrt{\left\langle\Phi^{\prime}(\eta(t, u)), W(\eta(t, u))\right\rangle} \mathrm{d} t \\
& \leq 2 \sqrt{t_{2}-t_{1}}\left[\int_{t_{1}}^{t_{2}}\left\langle\Phi^{\prime}(\eta(t, u)), W(\eta(t, u))\right\rangle \mathrm{d} t\right]^{1 / 2} \\
& =2 \sqrt{t_{2}-t_{1}}\left[\Phi\left(\eta\left(t_{1}, u\right)\right)-\Phi\left(\eta\left(t_{2}, u\right)\right)\right]^{1 / 2} \\
& \leq 2 \sqrt{t_{2}-t_{1}}[\Phi(u)-\tau]^{1 / 2} .
\end{aligned}
$$

Since $T^{+}(u)<+\infty$, this implies that $\lim _{t \rightarrow T^{+}(u)} \eta(t, u)$ exists and then it must be a critical point of $\Phi$ (otherwise the trajectory $t \mapsto \eta(t, u)$ could be continued beyond $T^{+}(u)$ ).

Case (2). $T^{+}(u)=+\infty$. To prove that $\lim _{t \rightarrow T^{+}(u)} \eta(t, u)$ exists, it suffices to show that

$$
\text { for every } \varepsilon>0 \text {, there exists } t_{\varepsilon}>0 \text { such that }\left\|\eta\left(t_{\varepsilon}, u\right)-\eta(t, u)\right\|<\varepsilon, \quad \forall t \geq t_{\varepsilon} \text {. }
$$

We suppose by contradiction that (4.6) is false. Then there exist $0<\varepsilon_{0}<\frac{1}{2} \min \left\{\kappa, \sqrt{2} c_{0}\right\}$ and a sequence $\left\{t_{n}\right\} \subset[0,+\infty)$ such that

$$
0 \leq t_{1}<t_{2}<\cdots<t_{n} \rightarrow+\infty \quad \text { and } \quad\left\|\eta\left(t_{n+1}, u\right)-\eta\left(t_{n}, u\right)\right\|=\varepsilon_{0}, \quad \forall n \in \mathbb{N} .
$$


Choose the smallest $t_{n}^{1} \in\left(t_{n}, t_{n+1}\right)$ and $s_{n}^{1} \in\left[t_{n}, t_{n}^{1}\right]$ such that

$$
\left\|\eta\left(t_{n}^{1}, u\right)-\eta\left(t_{n}, u\right)\right\|=\frac{\varepsilon_{0}}{3} \quad \text { and } \quad\left\|\Phi^{\prime}\left(\eta\left(s_{n}^{1}, u\right)\right)\right\|=\min _{t \in\left[t_{n}, t_{n}^{1}\right]}\left\|\Phi^{\prime}(\eta(t, u))\right\|
$$

Then it follows from (4.3), (4.4), and (4.8) that

$$
\begin{aligned}
\frac{\varepsilon_{0}}{3} & =\left\|\eta\left(t_{n}^{1}, u\right)-\eta\left(t_{n}, u\right)\right\| \\
& \leq \int_{t_{n}}^{t_{n}^{1}}\|W(\eta(t, u))\| \mathrm{d} t \\
& \leq 2 \int_{t_{n}}^{t_{n}^{1}}\left\|\Phi^{\prime}(\eta(t, u))\right\| \mathrm{d} t \\
& \leq \frac{2}{\left\|\Phi^{\prime}\left(\eta\left(s_{n}^{1}, u\right)\right)\right\|} \int_{t_{n}}^{t_{n}^{1}}\left\|\Phi^{\prime}(\eta(t, u))\right\|^{2} \mathrm{~d} t \\
& \leq \frac{2}{\left\|\Phi^{\prime}\left(\eta\left(s_{n}^{1}, u\right)\right)\right\|} \int_{t_{n}}^{t_{n}^{1}}\left\langle\Phi^{\prime}(\eta(t, u)), W(\eta(t, u))\right\rangle \mathrm{d} t \\
& =\frac{2}{\left\|\Phi^{\prime}\left(\eta\left(s_{n}^{1}, u\right)\right)\right\|}\left[\Phi\left(\eta\left(t_{n}, u\right)\right)-\Phi\left(\eta\left(t_{n}^{1}, u\right)\right)\right] .
\end{aligned}
$$

Since $\Phi\left(\eta\left(t_{n}, u\right)\right)-\Phi\left(\eta\left(t_{n}^{1}, u\right)\right) \rightarrow 0$ as $n \rightarrow \infty$, the above implies that $\Phi^{\prime}\left(\eta\left(s_{n}^{1}, u\right)\right) \rightarrow$ 0 as $n \rightarrow \infty$. Similarly we find the largest $t_{n}^{2} \in\left(t_{n}, t_{n+1}\right)$ and $s_{n}^{2} \in\left[t_{n}^{2}, t_{n+1}\right]$ such that $\left\|\eta\left(t_{n+1}, u\right)-\eta\left(t_{n}^{2}, u\right)\right\|=\frac{\varepsilon_{0}}{3}$ and $\Phi^{\prime}\left(\eta\left(s_{n}^{2}, u\right)\right) \rightarrow 0$ as $n \rightarrow \infty$. Let $u_{n}^{1}:=\eta\left(s_{n}^{1}, u\right)$ and $u_{n}^{2}:=$ $\eta\left(s_{n}^{2}, u\right)$. Then $\left\{u_{n}^{1}\right\}$ and $\left\{u_{n}^{2}\right\}$ are two Palais-Smale sequences of $\Phi$ such that $\frac{\varepsilon_{0}}{3} \leq \| u_{n}^{1}-$ $u_{n}^{2} \| \leq 2 \varepsilon_{0}<\min \left\{\kappa, \sqrt{2} c_{0}\right\}$. This, however, contradicts Lemma 4.2, hence (4.6) is true. So $\lim _{t \rightarrow T^{+}(u)} \eta(t, u)$ exists, and it must be a critical point of $\Phi$.

In the following, for a subset $A \subset E$ and $\delta>0$, we put $U_{\delta}(A):=\{v \in E: \operatorname{dist}(v, A)<\delta\}$.

Lemma 4.4 Let $c \geq c_{0}$. Then, for every $\delta>0$, there exists $\varepsilon=\varepsilon(\delta)>0$ such that

(a) $\Phi_{c-\varepsilon}^{c+\varepsilon} \cap \mathcal{K}=\mathcal{K}_{c}$;

(b) $\lim _{t \rightarrow T^{+}(u)} \Phi(\eta(t, u))<c-\varepsilon$ for $u \in \Phi^{c+\varepsilon} \backslash U_{\delta}\left(\mathcal{K}_{c}\right)$.

Proof In view of (4.1) and the $\mathbb{Z}^{3}$-translation invariance for $\Phi, \Phi(\mathcal{K}):=\{\Phi(w): w \in \mathcal{K}\}$ is a finite set. Therefore, there exists $\hat{\varepsilon}>0$ such that (a) is satisfied for $\varepsilon \in(0, \hat{\varepsilon})$.

Without loss of generality, we may assume $U_{\delta}\left(\mathcal{K}_{c}\right) \subset \Phi^{c+1}$ and $\delta<\min \left\{\kappa, \sqrt{2} c_{0}\right\}$. Next we find $\varepsilon \in(0, \hat{\varepsilon})$ such that (b) holds. Let $u \in \Phi^{c+\hat{\varepsilon}} \backslash U_{\delta}\left(\mathcal{K}_{c}\right)$. Since $\Phi(\eta(t, u))$ is strictly decreasing on $t \in\left[0, T^{+}(u)\right)$, if $\Phi\left(\eta\left(t_{0}, u\right)\right) \leq c-\hat{\varepsilon}$ for some $t_{0} \in\left[0, T^{+}(u)\right)$, then $\lim _{t \rightarrow T^{+}(u)} \Phi(\eta(t, u))<c-\hat{\varepsilon}$. Thus, we only consider the case where $\Phi(\eta(t, u))>c-\hat{\varepsilon}$ for all $t \in\left[0, T^{+}(u)\right)$. In this case, it follows from Lemma 4.3 that $\lim _{t \rightarrow T^{+}(u)} \eta(t, u)$ exists and is a critical point of $\Phi$. Set

$$
\alpha:=\inf \left\{\left\|\Phi^{\prime}(w)\right\|: w \in U_{\delta}\left(\mathcal{K}_{c}\right) \backslash U_{\delta / 2}\left(\mathcal{K}_{c}\right)\right\}
$$

We claim that $\alpha>0$. Indeed, suppose by contradiction that there exists a sequence $\left\{u_{n}^{1}\right\} \subset$ $U_{\delta}\left(\mathcal{K}_{c}\right) \backslash U_{\delta / 2}\left(\mathcal{K}_{c}\right)$ such that $\Phi^{\prime}\left(u_{n}^{1}\right) \rightarrow 0$. Passing to a subsequence, using the finiteness 
condition (4.1) and the $\mathbb{Z}^{3}$-invariance of $\Phi$, we may assume $\left\{u_{n}^{1}\right\} \subset U_{\delta}\left(w_{0}\right) \backslash U_{\delta / 2}\left(w_{0}\right)$ for some $w_{0} \in \mathcal{K}_{c}$. Let $u_{n}^{2}=w_{0}$. Then

$$
\frac{\delta}{2} \leq \limsup _{n \rightarrow \infty}\left\|u_{n}^{1}-u_{n}^{2}\right\| \leq \delta<\min \left\{\kappa, \sqrt{2} c_{0}\right\}
$$

which contradicts Lemma 4.2. Hence $\alpha>0$.

Let $\beta:=\sup \left\{\left\|\Phi^{\prime}(w)\right\|: w \in U_{\delta}\left(\mathcal{K}_{c}\right) \backslash U_{\delta / 2}\left(\mathcal{K}_{c}\right)\right\}$ and $0<\varepsilon<\frac{\alpha^{2} \delta}{4 \beta}$. By Lemma 4.3 and (a), the only way (b) can fail is that $\eta(t, u) \rightarrow \tilde{w} \in \mathcal{K}_{c}$ as $t \rightarrow T^{+}(u)$ for some $u \in \Phi^{c+\varepsilon} \backslash U_{\delta}\left(\mathcal{K}_{c}\right)$. In this case we let

$$
\begin{aligned}
& t_{1}:=\sup \left\{t \in\left[0, T^{+}(u)\right): \eta(t, u) \notin U_{\delta}(\tilde{w})\right\} \quad \text { and } \\
& t_{2} \in\left\{t \in\left[0, T^{+}(u)\right): \eta(t, u) \in U_{\frac{\delta}{2}}(\tilde{w})\right\} .
\end{aligned}
$$

Then from (4.3), (4.4), and (4.10), we have

$$
\frac{\delta}{2} \leq\left\|\eta\left(t_{2}, u\right)-\eta\left(t_{1}, u\right)\right\| \leq \int_{t_{1}}^{t_{2}}\|W(\eta(t, u))\| \mathrm{d} t \leq 2 \int_{t_{1}}^{t_{2}}\left\|\Phi^{\prime}(\eta(t, u))\right\| \mathrm{d} t \leq 2 \beta\left(t_{2}-t_{1}\right)
$$

and

$$
\Phi\left(\eta\left(t_{2}, u\right)\right)-\Phi\left(\eta\left(t_{1}, u\right)\right) \leq-\int_{t_{1}}^{t_{2}}\left\|\Phi^{\prime}(\eta(t, u))\right\|^{2} \mathrm{~d} t \leq-\alpha^{2}\left(t_{2}-t_{1}\right) \leq-\frac{\alpha^{2} \delta}{4 \beta} .
$$

Hence $\lim _{t_{2} \rightarrow T^{+}(u)} \Phi\left(\eta\left(t_{2}, u\right)\right) \leq c+\varepsilon-\frac{\alpha^{2} \delta}{4 \beta}<c$, contrary to our assumption.

Proof of Theorem 1.2 For $j \in \mathbb{N}$, we consider the family $\Sigma_{j}$ of all closed and symmetric subsets $A \subset E \backslash\{0\}$ (i.e., $A=-A=\bar{A}$ ) with $\gamma(A) \geq j$, where $\gamma$ denotes the usual Krasnoselskii genus (see, e.g., $[27,30]$ ). Moreover, we consider the nondecreasing sequence of Lusternik-Schnirelman values for $\Phi$ defined by $c_{k}:=\left\{c \in \mathbb{R}: \gamma\left(\Phi^{c}\right) \geq k\right\}$ for $k \in \mathbb{N}$. We claim:

$$
\mathcal{K}_{c_{k}} \neq \emptyset \quad \text { and } \quad c_{k}<c_{k+1}, \quad k \in \mathbb{N} \text {. }
$$

To prove this, let $k \in \mathbb{N}$ and $c=c_{k}$. In view of Lemma 4.1, $\gamma\left(\mathcal{K}_{c}\right)=0$ if $\mathcal{K}_{c}=\emptyset$ or $\gamma\left(\mathcal{K}_{c}\right)=1$ if $\mathcal{K}_{c} \neq \emptyset$. By the continuity property of the genus, there exists $\delta>0$ such that $\gamma\left(U_{\delta}\left(\mathcal{K}_{c}\right)\right)=$ $\gamma\left(\mathcal{K}_{c}\right)$. Choose $\varepsilon=\varepsilon(\delta)>0$ such that the properties of Lemma 4.4 hold. Then, for every $u \in \Phi^{c+\varepsilon} \backslash U_{\delta}\left(\mathcal{K}_{c}\right), t_{u} \in\left[0, T^{+}(u)\right)$, where $t_{u}:=\inf \left\{t \in\left[0, T^{+}(u)\right): \Phi(\eta(t, u)) \leq c-\varepsilon\right\}$. Since $\eta(t, u)$ is odd with respect to $u$ and $\Phi$ is even, it implies that $t_{u}=t_{-u}$. Define a map

$$
h: \Phi^{c+\varepsilon} \backslash U_{\delta}\left(\mathcal{K}_{c}\right) \rightarrow \Phi^{c-\varepsilon}, \quad h(u)=\eta\left(t_{u}, u\right) .
$$

Then $h$ is odd and continuous. Hence $\gamma\left(\Phi^{c+\varepsilon} \backslash U_{\delta}\left(\mathcal{K}_{c}\right)\right) \leq \gamma\left(\Phi^{c-\varepsilon}\right) \leq k-1$ and therefore

$$
\gamma\left(\Phi^{c+\varepsilon}\right) \leq \gamma\left(\overline{U_{\delta}\left(\mathcal{K}_{c}\right)}\right)+k-1=\gamma\left(\mathcal{K}_{c}\right)+k-1
$$

The definition of $c=c_{k}$ and of $c_{k+1}$ implies that $\gamma\left(\mathcal{K}_{c}\right) \geq 1$ if $c_{k+1}>c_{k}$ and $\gamma\left(\mathcal{K}_{c}\right)>1$ if $c_{k+1}=c_{k}$. Since $\gamma(\mathcal{F})=\gamma\left(\mathcal{K}_{c}\right) \leq 1$, (4.11) follows. 
It follows now from (4.11) that there is an infinite sequence $\left\{u_{k}\right\}$ of pairs of geometrically distinct critical points of $\Phi$ with $\Phi\left(u_{k}\right)=c_{k}$, contrary to (4.1). The proof is finished.

\author{
Acknowledgements \\ The authors would like to thank the referees for their useful suggestions. \\ Funding \\ The authors are supported financially by the National Natural Science Foundation of China (No: 11501190) and Hunan \\ Provincial Natural Science Foundation (No: 2019JJ50146).
}

\title{
Availability of data and materials
}

Not applicable.

Competing interests

The authors declare that they have no competing interests.

\section{Authors' contributions}

The research was carried out in collaboration. All authors read and assured the final manuscript.

\section{Author details}

${ }^{1}$ School of Mathematics and Computing Sciences, Hunan University of Science and Technology, Xiangtan, P.R. China.

${ }^{2}$ School of Mathematics and Statistics, Central South University, Changsha, P.R. China.

\section{Publisher's Note}

Springer Nature remains neutral with regard to jurisdictional claims in published maps and institutional affiliations.

Received: 18 January 2019 Accepted: 22 March 2019 Published online: 29 March 2019

\section{References}

1. Ambrosetti, A., Ruiz, D.: Multiple bound states for the Schrödinger-Poisson problem. Commun. Contemp. Math. 10, 391-404 (2008)

2. Azzollini, A.: Concentration and compactness in nonlinear Schrödinger-Poisson system with a general nonlinearity. J. Differ. Equ. 249(7), 1746-1763 (2010)

3. Azzollini, A., d'Venia, P., Pomponio, A.: On the Schrödinger-Maxwell equations under the effect of a general nonlinear term. Ann. Inst. Henri Poincaré, Anal. Non Linéaire 27(2), 779-791 (2010)

4. Azzollini, A., Pomponio, A.: Ground state solutions for the nonlinear Schrödinger-Maxwell equations. J. Math. Anal. Appl. 345, 90-108 (2008)

5. Benci, V., Fortunato, D.: An eigenvalue problem for the Schrödinger-Maxwell equations. Topol. Methods Nonlinear Anal. 11, 283-293 (1998)

6. Benci, V., Fortunato, D.: Solitary waves of the nonlinear Klein-Gordon equation coupled with Maxwell equations. Rev. Math. Phys. 14, 409-420 (2002)

7. Benguria, R., Brezis, H., Lieb, E.H.: The Thomas-Fermi-von Weizsäcker theory of atoms and molecules. Commun. Math. Phys. 79, 167-180 (1981)

8. Catto, I., Lions, P.L.: Binding of atoms and stability of molecules in Hartree and Thomas-Fermi type theories. Part 1: a necessary and sufficient condition for the stability of general molecular system. Commun. Partial Differ. Equ. 17, $1051-1110$ (1992)

9. Chen, S.T., Tang, X.H.: Ground state sign-changing solutions for a class of Schrödinger-Poisson type problems in $\mathbb{R}^{3}$. Z. Angew. Math. Phys. 67(4), 1-18 (2016)

10. Chen, S.T., Tang, X.H.: Nehari type ground state solutions for asymptotically periodic Schrödinger-Poisson systems. Taiwan. J. Math. 21, 363-383 (2017)

11. Chen, S.T., Tang, X.H., Liao, F.F.: Existence and asymptotic behavior of sign-changing solutions for fractional Kirchhoff-type problems in low dimensions. NoDEA Nonlinear Differ. Equ. Appl. 25, 40 (2018)

12. Chen, S.T., Tang, X.H.: Geometrically distinct solutions for Klein-Gordon-Maxwell systems with super-linear nonlinearities. Appl. Math. Lett. 90, 188-193 (2019)

13. Chen, S.T., Tang, X.H.: Ground state solutions of Schrödinger-Poisson systems with variable potential and convolution nonlinearity. J. Math. Anal. Appl. 473, 87-111 (2019)

14. D’Aprile, T., Mugnai, D.: Solitary waves for nonlinear Klein-Gordon-Maxwell and Schrödinger-Maxwell equations. Proc. R. Soc. Edinb., Sect. A 134, 1-14 (2004)

15. d'Avenia, P.: Non-radially symmetric solutions of nonlinear Schrödinger equation coupled with Maxwell equations. Adv. Nonlinear Stud. 2(2), 177-192 (2002)

16. He, X.M.: Multiplicity and concentration of positive solutions for the Schrödinger-Poisson equations. Z. Angew. Math Phys. 5, 869-889 (2011)

17. He, X.M., Zou, W.M.: Existence and concentration of ground states for Schrödinger-Poisson equations with critical growth. J. Math. Phys. 53, 023702 (2012)

18. He, X.M., Zou, W.M.: Multiplicity of concentrating positive solutions for Schrödinger-Poisson equations with critical growth. Nonlinear Anal. 170, 142-170 (2018)

19. Li, W., Radulescu, V.D., Zhang, B.L.: Infinitely many solutions for fractional Kirchhoff-Schrödinger-Poisson systems. J. Math. Phys. 60(1), 011506 (2019)

20. Lieb, E.H.: Thomas-Fermi and related theories and molecules. Rev. Mod. Phys. 53, 603-641 (1981) 
21. Lieb, E.H.: Sharp constants in the Hardy-Littlewood-Sobolev inequality and related inequalities. Ann. Math. 118, 349-374 (1983)

22. Lieb, E.H., Loss, M.: Analysis, Graduate Studies in Mathematics, vol. 14. AMS (1997)

23. Lions, P.L.: Solutions of Hartree-Fock equations for Coulomb systems. Commun. Math. Phys. 109, 33-97 (1984)

24. Lions, P.L.: The concentration-compactness principle in the calculus of variations. The locally compact case. I \& II. Ann. Inst. Henri Poincaré, Anal. Non Linéaire 1, 109-145, 223-283 (1984)

25. Mugnai, D.: The Schrödinger-Poisson system with positive potential. Commun. Partial Differ. Equ. 36, 1099-1117 (2011)

26. Papageorgiou, N.S., Radulescu, V.D., Repovs, D.D.: Nonlinear Analysis—Theory and Methods. Springer Monographs in Mathematics. Springer, Berlin (2019)

27. Rabinowitz, P.H.: Minimax Methods in Critical Point Theory with Applications to Differential Equations. CBMS Reg Conf. Ser. Math., vol. 65. Am. Math. Soc., Providence (1986)

28. Ruiz, D.: The Schrödinger-Poisson equation under the effect of a nonlinear local term. J. Funct. Anal. 237, 655-674 (2006)

29. Ruiz, D.: On the Schrödinger-Poisson-Slater system: behavior of minimizers, radial and nonradial cases. Arch. Ration. Mech. Anal. 198, 349-368 (2010)

30. Struwe, M.: Variational Methods, 2nd edn. Springer, Berlin (1996)

31. Sun, J.J., Ma, S.W.: Ground state solutions for some Schrödinger-Poisson systems with periodic potentials. J. Differ. Equ. 260, 2119-2149 (2016)

32. Szulkin, A., Weth, T.: Ground state solutions for some indefinite variational problems. J. Funct. Anal. 257, 3802-3822 (2009)

33. Tang, X.H.: Non-Nehari manifold method for superlinear Schrodinger equation. Taiwan. J. Math. 18(6), 1957-1979 (2014)

34. Tang, X.H.: Non-Nehari manifold method for asymptotically linear Schrödinger equation. J. Aust. Math. Soc. 98, 104-116 (2015)

35. Tang, X.H.: Non-Nehari manifold method for asymptotically periodic Schrödinger equation. Sci. China Math. 58, 715-728 (2015)

36. Tang, X.H., Chen, S.T.: Ground state solutions of Nehari-Pohožaev type for Schrödinger-Poisson problems with general potentials. Discrete Contin. Dyn. Syst. 37, 4973-5002 (2017)

37. Willem, M.: Minimax Theorems. Birkhäuser, Boston (1996)

38. Xiang, M.Q., Wang, F.L.: Fractional Schrödinger-Poisson-Kirchhoff type systems involving critical nonlinearities. Nonlinear Anal. 164, 1-26 (2017)

39. Xiang, M.Q., Radulescu, V.D., Zhang, B.L.: Combined effects for fractional Schrödinger-Kirchhoff systems with critical nonlinearities. ESAIM Control Optim. Calc. Var. 24(3), 1249-1273 (2018)

40. Zhang, X., Zhang, B.L., Repovs, D.: Existence and symmetry of solutions for critical fractional Schrodinger equations with bounded potentials. Nonlinear Anal. 142, 48-68 (2016)

41. Zhao, L.G., Zhao, F.K.: On the existence of solutions for the Schrödinger-Poisson equations. J. Math. Anal. Appl. 346, 155-169 (2008)

\section{Submit your manuscript to a SpringerOpen ${ }^{\circ}$ journal and benefit from:}

- Convenient online submission

- Rigorous peer review

- Open access: articles freely available online

- High visibility within the field

- Retaining the copyright to your article

Submit your next manuscript at $\boldsymbol{\nabla}$ springeropen.com 